\title{
The effect of hypoxic microenvironment on matrix metalloproteinase expression in xenografts of human oral squamous cell carcinoma
}

\author{
YASUO MIYAZAKI $^{1}$, AKIRA HARA $^{2}$, KEIZO KATO $^{1}$, TAKERU OYAMA $^{2}$, \\ YASUHIRO YAMADA ${ }^{2}$, HIDEKI MORI ${ }^{2}$ and TOSHIYUKI SHIBATA ${ }^{1}$ \\ ${ }^{1}$ Department of Oral and Maxillofacial Sciences and ${ }^{2}$ Department of Tumor Pathology, \\ Graduate School of Medicine, Gifu University, 1-1 Yanagido, Gifu 501-1194, Japan
}

Received August 3, 2007; Accepted September 7, 2007

\begin{abstract}
Hypoxia is a common environmental stress factor and is associated with physiological and pathological conditions related to cancer invasion and metastasis. The process of cancer cell invasion involves degradation of the extracellular matrix. Here, we examine the effect of hypoxic microenvironment on matrix metalloproteinase expression in human oral squamous cell carcinoma under in vitro and in vivo conditions. At first, the expression levels of HIF-1 $\alpha$ and matrix metalloproteinase (MMP) proteins in human oral squamous cell carcinoma cell lines, SAS and HSC-2 cultured under hypoxic or normoxic condition, were assessed by Western blotting. Enzyme activity and mRNA of MMP under hypoxic or normoxic condition were also investigated. Then the SAS and HSC-2 cells were transplanted subcutaneously into immunodeficient mice and the correlation between hypoxia and protein expression for MMPs, HIF- $1 \alpha$ and $\mathrm{Ki}-67$ were assessed. Hypoxic region was detected by in situ hypoxic probe, pimonidazole. MMP proteins and mRNA in both SAS and HSC-2 cells were increased under hypoxic condition. In xenograft, MMP-2 was expressed in tumor tissue, especially in hypoxic region. In contrast, MMP-9 expression was recognized in tumor tissue, especially neighboring stromal tissues containing blood vessels. Our study suggests that the hypoxic microenvironment in human oral squamous cell carcinoma plays important roles in expression for HIF- $1 \alpha$ and MMPs, and proliferative activity of tumor cells.
\end{abstract}

Correspondence to: Dr Akira Hara, Department of Tumor Pathology, Graduate School of Medicine, Gifu University, 1-1 Yanagido, Gifu 501-1194, Japan

E-mail: ahara@gifu-u.ac.jp

Key words: matrix metalloproteinase, HIF-1 $\alpha$, pimonidazole, hypoxia, oral squamous cell carcinoma

\section{Introduction}

Oral squamous cell carcinoma (OSCC) is the most common head and neck cancer and the overall prognosis of patients with this remains poor. Although extensive research has being done on the pathogenesis and management, the 5-year survival rate for patients with OSCC has not improved within the last 25 years, and it remains at $53 \%$ (1). The poor prognosis of OSCC is caused by its local invasion and distant metastasis $(2,3)$.

The pathological features such as active invasiveness, lymph node metastasis and vascular proliferation are caused by tumor tissue hypoxia. Hypoxia is a common feature of many cancers. Hypoxia is speculated to contribute to local and systemic tumor progression as well as potentially compromising radiotherapy and chemotherapy (4). Under hypoxic conditions, hypoxia-inducible factor-1 (HIF-1) is a crucial transcriptional factor in the cellular response to hypoxia (5). HIF-1 activates the transcription of many genes that code for proteins involved in embryogenesis, angiogenesis, glucose metabolism, cell proliferation and invasiveness (6). Indeed overexpression of HIF-1 has been demonstrated in many human tumors and predicts a poor patient survival (7). Molecular mechanism of HIF-1 has been clarified in various fields of investigation (8), however, its contributory roles of tumor microenvironment under hypoxia are still unknown.

In tumor microenvironment, it was difficult to measure the tissue oxygen gradients at the cell level until recently. The immunohistochemical technique using a hypoxia marker, pimonidazole is a very interesting method because gradients of hypoxia can be visualized in situ. For example, spatial relationship between tumor vasculature and pimonidazole binding was studied in a human glioma xenograft model with a multiple staining and functional microscopic imaging technique (9). The density of the perfused vessels was inversely related to the pimonidazole hypoxic fraction. Furthermore a clinical study by Kaanders and colleagues on biopsies from patients with squamous cell carcinomas (SCC) of head and neck indicated a prognostic value of pimonidazole for local control (10). This means histological analysis of hypoxic environment in SCC provides important clinical information on patient prognosis. 
For tumor invasion and metastasis, matrix metalloproteinases (MMPs), especially MMP-2 and MMP-9 which are known as gelatinase $\mathrm{A}$ and $\mathrm{B}$, respectively, play a critical role to degradate of extracellular matrix (ECM) components (11). However, the molecular relationship between MMP induction and the activation of HIF-1 under hypoxic condition are still controversial (12). Thus the analysis for HIF-1 and MMP family under hypoxic tumor microenvironment using in situ hypoxic probe provides important information on tumor invasion and clinical therapy implication.

In the present study, we investigated the increased expression of HIF-1 and MMPs in human SCC cell lines under hypoxic conditions in vitro, and then we prepared tumor xenografts from the same human SCC cells and exposed tumor-bearing mice to the hypoxia probe pimonidazole. The visualized gradients of hypoxia were compared with the expression and the localization of HIF-1 $\alpha$, Ki-67 that indicates the cell proliferation, and MMPs in human SCC cells growing in nude mice.

\section{Materials and methods}

Reagents. The following antibodies were obtained from Daiichi Fine Chemical Co., Ltd. (Toyama, Japan): mouse monoclonal anti-hMMP-2 antibody (catalog no. F-68, human antigen), mouse monoclonal anti-hMMP9 antibody (catalog no. F-69, human antigen) and mouse monoclonal anti-hMT1-MMP antibody (catalog no. F-84, human antigen). A mouse monoclonal anti-HIF- $1 \alpha$ antibody was obtained from Novus Biologicals, Inc. (Littleton, CO; catalog no. NB100-105, human antigen), and a monoclonal mouse anti-Ki-67 antibody was from DacoCytomation (Glostrup, Denmark; catalog no. M 7240). A mouse monoclonal antibody against Actin (C-2) was purchased from Santa Cruz Biotechnology, Inc. (Santa Cruz, CA; catalog no. sc-8432). Hypoxiprobe ${ }^{\mathrm{TM}}-1$ including pimonidazole hydrochloride and anti-pimonidazole antibody (Hypoxiprobe-1 Mab1) was obtained from Chemicon International, Inc. (Temecula, CA; catalog no. HP1-100).

Cell culture and hypoxic treatment. Two cell lines, SAS and HSC2 were used in the present study. SAS was established from poorly differentiated human OSCC and HSC2 was from well-differentiated human OSCC. They were cultured in RPMI-1640 medium (Sigma-Aldrich Co., St. Louis, MO) supplemented with $10 \%$ FBS (Sigma), $100 \mathrm{U} / \mathrm{ml}$ penicillin and $100 \mu \mathrm{g} / \mathrm{ml}$ streptomycin (Sigma) at $37^{\circ} \mathrm{C}, 5 \% \mathrm{CO}_{2}$. For hypoxic culture, AnaeroPac ${ }^{\circledR}$ KENKI for Cells (Mitsubishi Gas Chemical Company, Inc., Tokyo, Japan) containing $\mathrm{O}_{2}$ absorbing and $\mathrm{CO}_{2}$ generating agents was used with its exclusive jar. It made the condition $<1 \% \mathrm{O}_{2}$ and $\sim 5 \% \mathrm{CO}_{2}$. Confluent cultured plates $(80 \%)$ were incubated in the jar at $37^{\circ} \mathrm{C}$ for $24 \mathrm{~h}$. Control cultures were incubated under normoxia ( $21 \%$ oxygen tension).

Western blot analysis. Cells cultured in hypoxia or normoxia were lysed for Western blot analysis. After centrifugation, the protein content was determined by fluorescence method. $20 \mu \mathrm{g}$ total protein of each samples were fractionated $10 \%$ SDS-PAGE gel and transferred onto Immobilon-P PVDF membranes (Millipore Corp., Bedford, MA). The membranes were blocked with $7 \%$ skimmed milk and then incubated with antibodies against HIF-1 $\alpha$ (1:500 dilution), MMP-2 (1:500 dilution), MMP-9 (1:500 dilution), MT1-MMP (1:500 dilution), or actin (1:1000 dilution) at $4^{\circ} \mathrm{C}$ overnight. After washing with $\mathrm{PBS}$, they were incubated with peroxidaseconjugated goat anti-mouse IgG antibody (1:1000 dilution; Dako). Chemiluminescence reagents (ECL Western Blot Analysis Detection Reagents; GE Healthcare, Piscataway, $\mathrm{NJ}$ ) were used to visualize the labeled protein bands.

Gelatin zymography. SAS and HSC-2 cells were cultured in serum-free media for $24 \mathrm{~h}$ under hypoxic or normoxic condition, and conditioned media was concentrated using Amicon ${ }^{\circledR}$ Ultra centrifugal filter devices (Millipore) as per manufacturer's instruction. An equal volume of sample buffer (63 mM Tris- $\mathrm{HCl}$, pH 6.8, 10\% glycerol, 2\% SDS, and $0.0025 \%$ bromophenol blue; TEFCO, Tokyo, Japan) was added to the concentrated media before being applied to a $10 \%$ SDS-PAGE gel impregnated with $0.1 \%$ gelatin. After electrophoresis the gel was rinsed with renaturing buffer (TEFCO) for $30 \mathrm{~min}$, and incubated in developing buffer (TEFCO) for $24 \mathrm{~h}$ at $37^{\circ} \mathrm{C}$. After incubation, the gel was stained with $0.05 \%$ Coomassie brilliant blue R-250 (Wako Pure Chemicai Industries, Osaka, Japan). MMPs were detected as transparent bands. Human proMMP-2 (Wako; catalog no. 133-12631) and human proMMP-9 (Wako; catalog no. 130-12641) were used as a positive control.

$R T$-PCR and quantitative real-time PCR. Total cellular RNA was purified using the TRIzol Reagent ${ }^{\circledR}$ (Invitrogen, Carlsbad, CA). Total RNA concentration was measured by GeneQuant (GE Healthcare). Reverse transcription was carried out by SuperScript $^{\mathrm{TM}}$ III First-Strand Synthesis System for RT-PCR (Invitrogen) according to the manufacturer's instruction, and then, real-time RT-PCR assay was performed $\left(\mathrm{SYBR}^{\circledR}\right.$ Premix Ex Taq ${ }^{\mathrm{TM}}$, with Thermal Cycler Dice ${ }^{\circledR}$; Takara, Tokyo, Japan). Primer sequence was shown (Table I). The cycling conditions were initial denaturation at $95^{\circ} \mathrm{C}$ for $10 \mathrm{sec}$, and 45 cycles at $95^{\circ} \mathrm{C}$ for $10 \mathrm{sec}, 60^{\circ} \mathrm{C}$ for $10 \mathrm{sec}$, and $72^{\circ} \mathrm{C}$ for $8 \mathrm{sec}$. Second derivative maximum method was used to calculate the $\mathrm{C}_{\mathrm{t}}$ (thershold cycle) value and standard curve method was used for relative quantification analysis. The $\mathrm{C}_{\mathrm{t}}$ values of MMP-2 and MMP-9 were normalized by the $C_{t}$ of GAPDH in the same sample.

Preparation of xenografts of human OSCC. Male nude (BALB/c-nu/nu Slc) mice were purchased from CLEA Japan, Inc. (CLEA, Tokyo, Japan). Four mice aged 7-8 weeks were used for the xenografts experiment. SAS cells $\left(1 \times 10^{7}\right)$ were injected subcutaneously into the flanks of 2 of 4 mice, and HSC-2 cells $\left(1 \times 10^{7}\right)$ into the other mice. Four weeks after the cell transplantation, we injected pimonidazole hydrochloride intravenously at a dose of $60 \mathrm{mg} / \mathrm{kg}$ body weight to detect oxygen gradients in xenografts, and then mice were sacrificed $1 \mathrm{~h}$ after the injection. Tumors with neighboring skeletal muscles were harvested, fixed with formalin and embedded in paraffin. We fully complied with the 'Guidelines Concerning Experimental Animals' issued by the Japanese Association for Laboratory Animal Science and exercised due consideration to the ethics relating to animal studies. 
Table I. Primer sequences.

\begin{tabular}{|c|c|c|}
\hline Target molecules & & Primer sequences \\
\hline MMP-2 & $\begin{array}{l}\text { Forward } \\
\text { Reverse }\end{array}$ & $\begin{array}{l}\text { 5'-CCССТCCAATCCCACCAAC-3' } \\
\text { 5'-TCCATTGGCTCCAAGGGTTC-3' }\end{array}$ \\
\hline MMP-9 & $\begin{array}{l}\text { Forward } \\
\text { Reverse }\end{array}$ & $\begin{array}{l}\text { 5'-TCTGCCCGGACCAAGGATAC-3' } \\
\text { 5'-GGTTCAGGGCGAGGACCATA-3' }\end{array}$ \\
\hline GAPDH & $\begin{array}{l}\text { Forward } \\
\text { Reverse }\end{array}$ & $\begin{array}{l}\text { 5'-TGGTATCGTGGAAGGACTCATGAC-3' } \\
\text { 5'-ATGCCAGTGAGCTTCCCGTTCAGC-3' }\end{array}$ \\
\hline
\end{tabular}
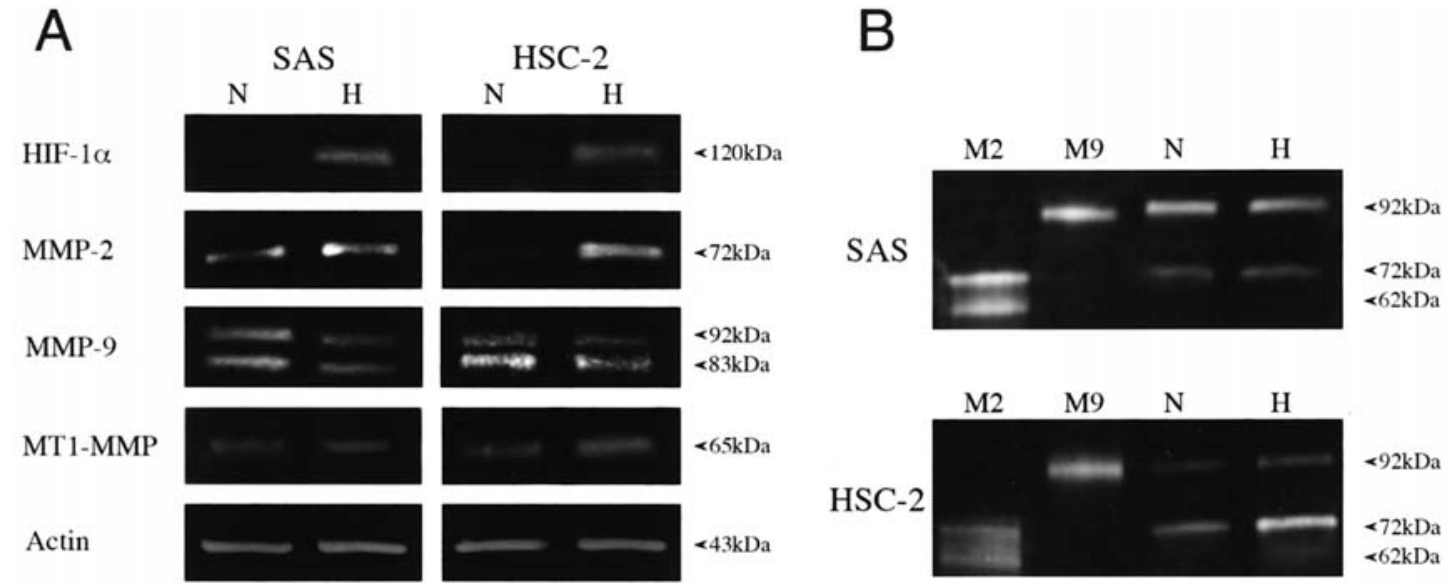

Figure 1. Effect of hypoxia on SAS and HSC-2 cell lines in Western blot analysis and gelatin zymography. A, Whole cell lysates of SAS and HSC-2 cells were immunoblotted with antibodies for HIF-1 $\alpha$, MMP-2, MMP-9 or MT1-MMP under conditions of normoxia (N) or hypoxia (H) for 24 h. B, The gelatinolytic activity of the MMP-2 and MMP-9 under conditions of normoxia (N) or hypoxia $(\mathrm{H})$ for $24 \mathrm{~h}$ was measured by gelatin zymography. Aliquots of appropriately condensed conditioned media from SAS and HSC-2 cells were loaded on gelatin gels. Purified proMMP-2 (M2) and proMMP-9 (M9) enzymes are shown as positive control.

Hematoxylin and eosin $(H \& E)$, victoria blue, and immunohistochemical staining of histological sections. Serial sections (3- $\mu$ m thick) were cut from each paraffin-embedded block. One section was stained with H\&E for histological assessment, and another one was stained with victoria blue (VB) staining to confirm the interstitium. Other sections were immunochemically stained for pimonidazole hydrochloride, HIF-1 $\alpha$, Ki-67, MMP-2, MMP-9, or MT1-MMP. Sections were deparaffinized, rehydrated through a graded alcohol series, and heated in 10-mM sodium citrate ( $\mathrm{pH}$ 6.0) for $5 \mathrm{~min}$ in a microwave to retrieve the antigens, and then we used Mouse To Mouse HRP (ScyTec Lab., UT, USA) according to the instructions supplied by the manufacturer. The primary antibodies against pimonidazole hydrochloride (1:200 dilution), HIF-1 $\alpha$ (1:250 dilution), Ki-67 (1:1000 dilution), MMP-2 (1:500 dilution), MMP-9 (1:500 dilution), and MT1-MMP (1:100 dilution) were applied for $8 \mathrm{~h}$ at $4^{\circ} \mathrm{C}$. After washing with PBS, diaminobenzidine solution (Dako) was applied. Nuclei were counterstained with hematoxylin.

\section{Results}

Effect of hypoxia on HIF-1 $\alpha$ and MMPs protein expressions. The effect of hypoxia on intracellular expressions of HIF-1 $\alpha$ and MMP proteins were analyzed using Western blot analysis
(Fig. 1A). The up-regulated expression of HIF-1 $\alpha$ and MMP-2 proteins in human SCC cell lines, SAS and HSC-2 was recognized under hypoxic conditions for $24 \mathrm{~h}$. Expression of MMP-9 and MT1-MMP proteins, however, were not increased in the cell lines. In gelatin zymography, gelatinolytic activity was confirmed in the MMP-2 and MMP-9 proteins released into culture medium by both cell lines (Fig. 1B). Purified pro- and active enzymes for MMP-2 and MMP-9 are shown as reference. MMP enzyme activity for gelatin degradation was more notable in MMP-2 than MMP-9 in both cell lines.

Expression of MMP-2 and MMP-9 mRNA in hypoxia. Quantitative real-time RT-PCR analysis of MMP-2 and MMP-9 expression performed on SAS and HSC cells cultured under conditions of normoxia or hypoxia for $24 \mathrm{~h}$ showed significant differences. The expression of MMP-2 of both SAS and HSC2 cells cultured under hypoxia was significantly increased compared to cells cultured under normoxia (SAS: $\mathrm{P}=0.0006$, HSC2: $\mathrm{P}=0.0057$; Fig. 2A). The MMP-9 mRNA levels in these cells were significantly up-regulated by hypoxia (SAS: $\mathrm{P}=0.0053$, HSC2: $\mathrm{P}=0.0071$; Fig. 2B).

Histopathological findings in OSCC cell lines growing in nude mice. The tumor growth of transplanted SAS and 
A
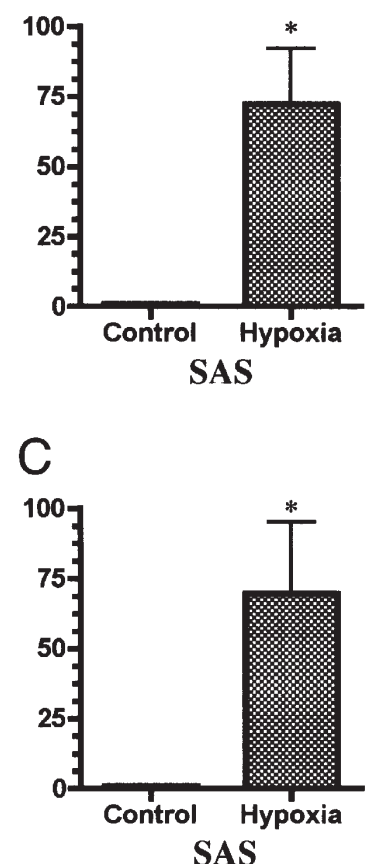

$\mathrm{B}$
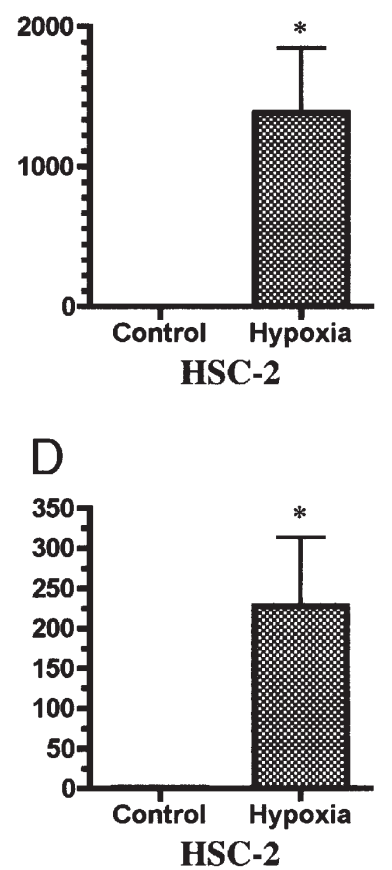

Figure 2. Real-time quantitative RT-PCR results (measured by gene/GAPDH). The effect of hypoxia on MMP-2 (A) and MMP-9 (B) mRNA expression in SAS and HSC-2 cells were detected by real-time quantitative RT-PCR. SAS and HSC-2 cells were cultured under conditions of normoxia or hypoxia for $24 \mathrm{~h}$. The results were scaled by dividing each set of results by the value of normoxia. ${ }^{*} \mathrm{P}<0.01$ vs. normoxia control, $\mathrm{n}=4$.

HSC-2 cells in nude mice at 4 weeks after transplantation was observed in $8 / 8$ (tumors/injections) in two mice and $8 / 8$ in two mice, respectively. By a conventional HE staining, the transplanted SAS cells showed aggressive invasion into interstitial tissues and poorly differentiated morphology of SCC (Fig. 3A). The transplanted HSC-2 cells showed aggressive invasion into interstitial tissues and well differentiated morphology of SCC associated with keratosis (Fig. 4A). These tumors resembled human OSCC morphologically. The distribution of HIF- $1 \alpha$ and MMP-2 immunostaining within tumor nest of SAS cell line xenografts is consistent with that of intra-nuclear staining of pimonidazole (Fig. 3C, E and $\mathrm{B}$, respectively). The expression of MMP-9, however, was detected within the tumor nest with stronger expression in tumor cells adjacent to interstitial tissues (Fig. 3F). The MT1-MMP was distributed homogeneously within the tumor nest (Fig. 3G). The HSC-2 xenografts showed almost similar histological findings to SAS xenografts (Fig. 4B, C, E, F and $\mathrm{G})$. To compare the tissue oxygen gradients and tumor cell growth rates, Ki-67 labelling was performed in both xenografts. Within tumor tissues, intra-nuclear accumulation of pimonidazole and Ki-67 nuclear staining were inversely correlated in both xenografts (Figs. 3B and D, and 4B and D, respectively). Victoria blue staining demonstrated interstitial tissues (Figs. 3H and 4H).

\section{Discussion}

The expression of hypoxia-regulated genes that increase glycolysis, cell survival and oxygen delivery promotes an

aggressive tumor phenotype and is associated with an adverse cancer treatment outcome. Several transcription factors have been reported participating in regulating gene expression of mammalian cells under hypoxic conditions (13). In the present study, the in vitro study demonstrated the increased expression of HIF- $1 \alpha$ and MMP-2 proteins in human SCC cell lines, SAS and HSC-2 under hypoxic conditions for $24 \mathrm{~h}$. Expression of MMP-9 protein, however, was not increased in the cell lines. The result of the expression of MMP-2 and MMP-9 mRNA was slightly different from that of the Western blot analysis. MMP-2 and MMP-9 mRNA in both cell lines under hypoxic conditions for $24 \mathrm{~h}$ were significantly increased. This indicates that for mRNA of MMP-2 and MMP-9, $24 \mathrm{~h}$ is long enough for increasing response to hypoxic stress. The MMP-2 and MMP-9 proteins released into culture medium were confirmed active by zymography. Similar to Western blot analysis, MMP-2 is more critical to enzyme activity for gelatin degradation than MMP-9. The results of our in vitro study suggest that MMP-2 plays more important roles than MMP-9 in human SCC cells under hypoxic conditions.

The mice bearing xenografts from the same human SCC cell lines were exposed to the hypoxia probe, pimonidazole. In tumor microenvironment, it was difficult to measure the tissue oxygen gradients at the cell level until recently. However, pimonidazole, one of 2-nitroimidazoles, has made this possible $(9,14)$. Although there are many methods detecting the hypoxia using various exogenous and endogenous markers (14), immunohistochemical method using an anti-pimonidazole antibody against pimonidazole is exceptionally attractive. Intranuclear accumulation of pimonidazole adducts strongly increases at $\mathrm{pO}_{2}$ levels $<10 \mathrm{~mm} \mathrm{Hg}$ (15). The advantage of this method is that the oxygen gradients within tumor tissues can be visualized and compared with hypoxia-related protein expression on a cell-by-cell basis.

For tumor invasion and metastasis, MMPs, especially MMP-2 and MMP-9, play a critical role in degradation of extracellular matrix (ECM) components $(11,16)$. Under hypoxic condition, invasion of hepatocellular carcinoma is accelerated by up-regulating the MMP family (12) and breast cancer invasion under hypoxic tumor microenvironments is promoted through an MT1-MMP-dependent mechanism (17). In the present study, the visualized gradients of hypoxia are compared with the expression and the localization of HIF-1 $\alpha, \mathrm{Ki}-67$ that indicates the cell proliferation, and MMPs including MMP-2, MMP-9 and MT1-MMP in human SCC cells growing in nude mice. In both xenografts derived from HSC-2 and SAS cell lines, the distribution of HIF-1 $\alpha$ and MMP-2 immunostaining within tumor nest is consistent with the intra-nuclear accumulation of pimonidazole. Thus, the expression of HIF-1 $\alpha$ and MMP-2 is inversely correlated to tissue oxygen gradients. The expression of MMP-9, however, was detected within the tumor nest with stronger expression in tumor cells adjacent to interstitial tissues. The MT1-MMP was distributed homogeneously within the tumor nest. Similar to the in vitro study, the results suggest that MMP-2 expression is influenced much more by hypoxia within tumor tissues than MMP-9 in xenografts.

On the other hand, HIF-1 is an essential regulator of oxygen homeostasis $(5,18)$. HIF-1 is a heterodimeric protein complex composed of two subunits, HIF- $1 \alpha$ and HIF-1ß. The 


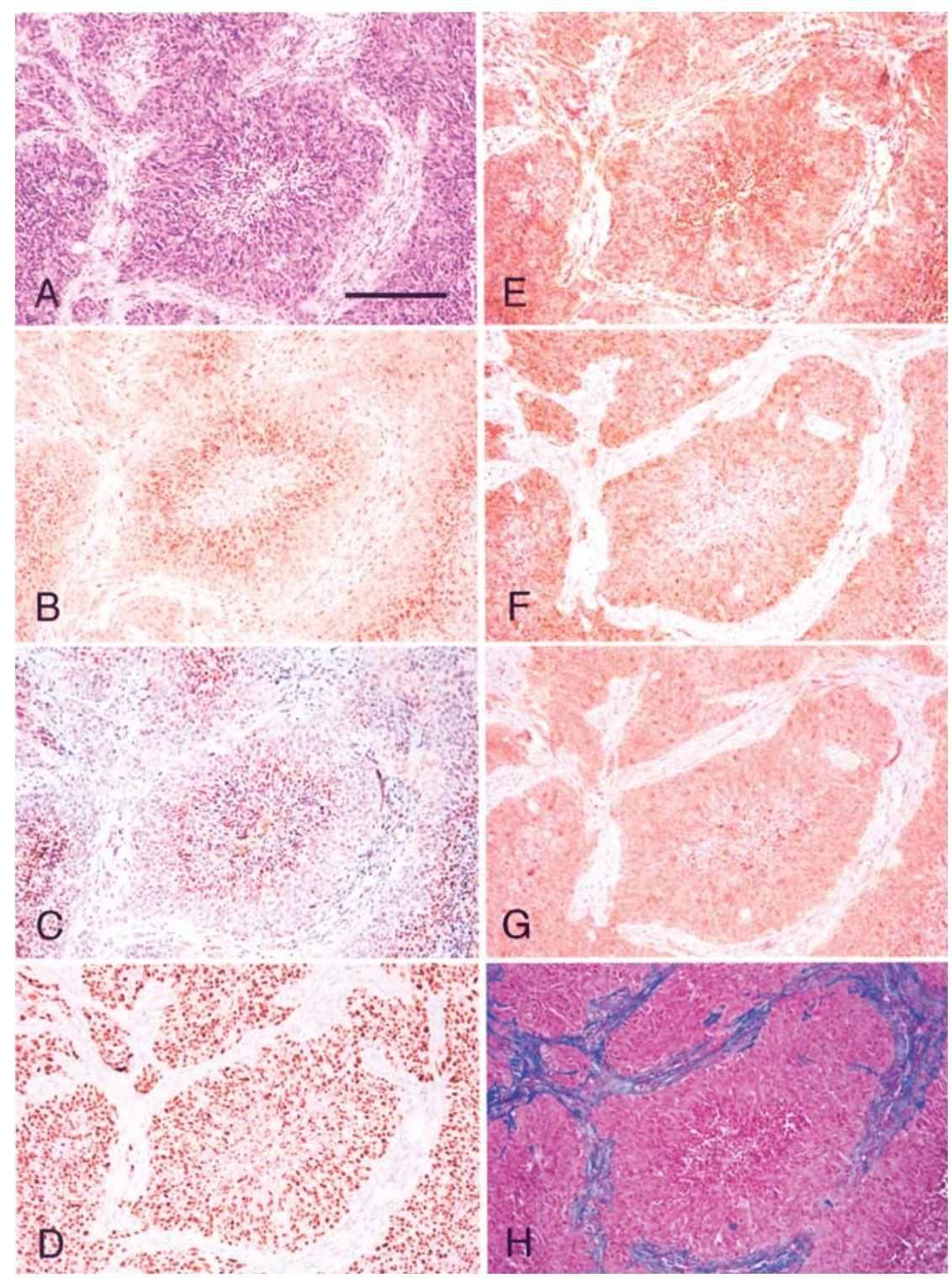

Figure 3. Representative microphotographs of the human squamous cell carcinoma cell line SAS growing in nude mice. Four weeks after the cell transplantation, pimonidazole hydrochloride was injected intravenously at a dose of $60 \mathrm{mg} / \mathrm{kg}$ body weight to detect oxygen gradients in xenografts, and then mice were sacrificed one hour after the injection. Formalin-fixed, paraffin-embedded tissues were obtained, and specimens were stained with following staining methods. A, Hematoxylin and eosin staining; B, Anti-pimonidazole immunostaining; C, Anti-HIF-1 $\alpha$ immunostaining; D, Anti-Ki-67 immunostaining; E, Anti-MMP-2 immunostaining; F, Anti-MMP-9 immunostaining; G, Anti-MT1-MMP immunostaining; H, Victoria blue staining. Scale bar, $200 \mu \mathrm{m}$.

HIF-1ß protein is expressed constitutively, whereas HIF-1 $\alpha$ protein is not detectable in cells under normoxic conditions (18). In normoxia, HIF- $1 \alpha$ protein degradation is regulated by $\mathrm{O}_{2}$-dependent prolyl hydroxylation, which targets the protein for ubiquitylation by E3 ubiquitin-protein ligases containing the von Hippel-Lindau tumor-suppressor protein (VHL), which binds specifically to hydroxylated HIF-1 $\alpha$ (6). Then ubiquitylated HIF-1 $\alpha$ is rapidly degraded by the proteasome. On the contrary, under hypoxic conditions, proline hydroxylation is inhibited, thereby stabilizing HIF-1 $\alpha$, which can then translocate into the nucleus and bind to HIF-1ß, forming the active HIF-1 complex (6). Thus HIF- $1 \alpha$ is an oxygen-regulated subunit and controls the transcription of many genes involved in angiogenesis, glucose metabolism, cell proliferation and invasiveness.

However, the molecular relationship between MMP induction and the activation of HIF-1 under hypoxic condition are still controversial (16). In fact, Miyoshi et al reported that hypoxic stress accelerates cancer invasion by up-regulating MMP family by an HIF- $1 \alpha$-independent pathway (12). Contrary to this result, Fujiwara et al demonstrated evidence for strong relationship between HIF-1 and MMP in glioma cell lines. They revealed that treatment with HIF- $1 \alpha$ siRNA resulted in down-regulation of MMP-2 mRNA in all glioma cell lines under hypoxic condition (19). Thus, the analysis for HIF- $1 \alpha$ and MMP family under hypoxic tumor microenvironment using in situ hypoxic probe provides important information on tumor invasion and clinical therapy implication. Our results demonstrated a correlation between gradients of hypoxia and MMP-2 distribution within tumor tissues while MMP-9 and MT1-MMT are expressed without interrelation of oxygen gradients. The findings of hypoxiaassociated MMP-2 expression are supported by recent reports on expression of MMT family in human OSCC. Activated 


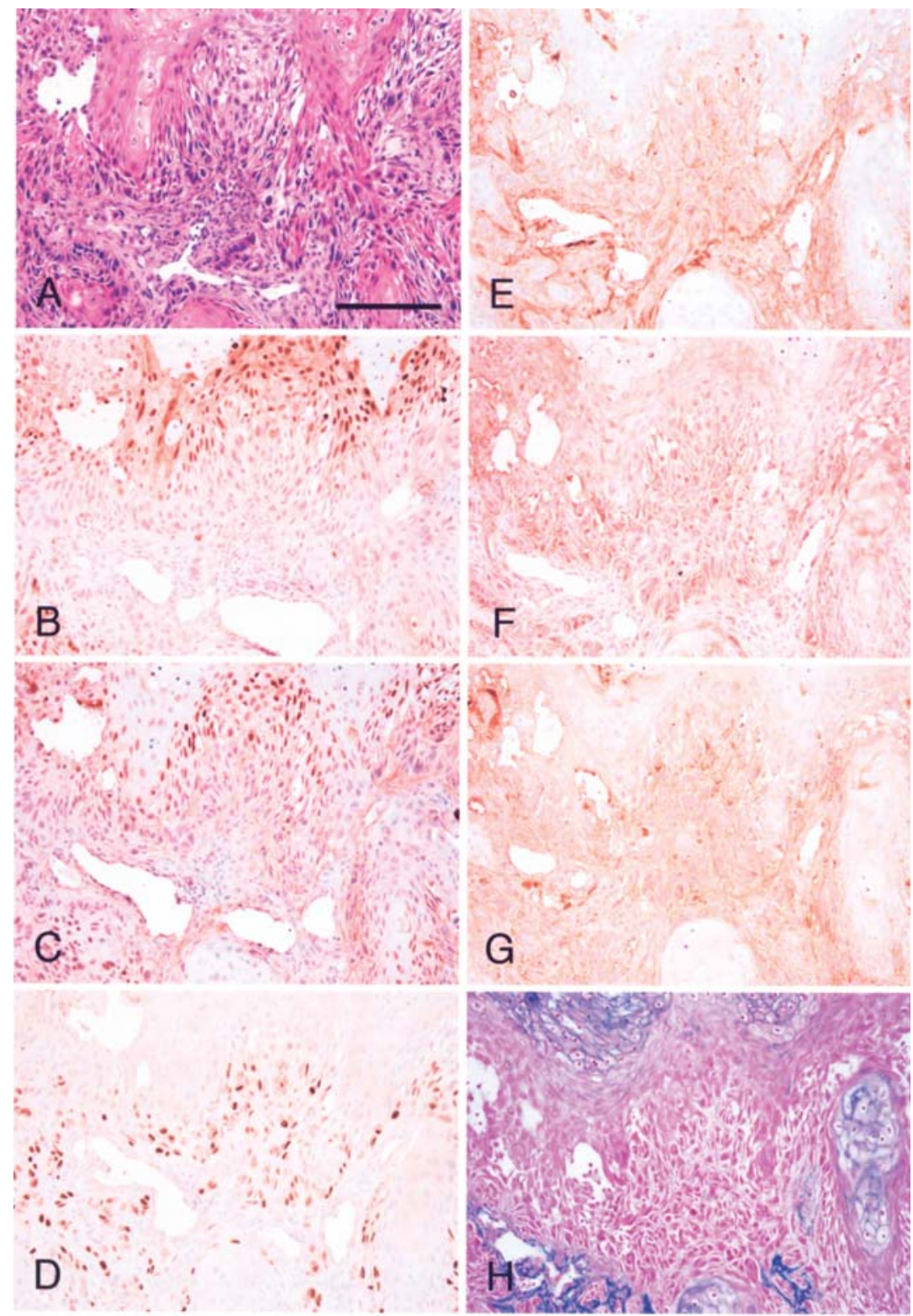

Figure 4. Representative microphotographs of the human squamous cell carcinoma cell line, HSC-2 growing in nude mice. A, Hematoxylin and eosin staining; B, Anti-pimonidazole immunostaining; C, Anti-HIF-1 $\alpha$ immunostaining; D, Anti-Ki-67 immunostaining; E, Anti-MMP-2 immunostaining; F, AntiMMP-9 immunostaining; G, Anti-MT1-MMP immunostaining; H, Victoria blue staining. Scale bar, $200 \mu \mathrm{m}$.

MMP-2 is assumed as a main enzymatic activity of ECM degradation by human OSCC $(20,21)$. Furthermore, our in vitro study also support MMP-2 dominancy under hypoxic microenvironment because the increased expression of HIF$1 \alpha$ and MMP-2 proteins was observed under hypoxic conditions for $24 \mathrm{~h}$ although expression of MMP-9 protein was not increased under similar conditions. In tumor microenvironment, the distribution of HIF-1 $\alpha$ and MMP-2 is consistent with oxygen gradients, however, tumor cell proliferative marker, Ki-67 nuclear staining was inversely correlated with oxygen gradients. This suggests that HIF-1 $\alpha$ and MMP-2 may act as a paracrine factor rather than an autocrine fashion in the tumor under hypoxic microenvironment.

In conclusion, immunohistochemical examination is not always suitable for the evaluation of MMP performance, since immunohistochemistry reflects both pro and activated forms of MMPs (20). Analysis of in situ hypoxic microenvironment elucidates the activated MMPs correlated with oxygen gradients and the interaction of MMPs between tumor cells and surrounding stromal cells in OSCC. Recent studies have revealed that tumor cells are capable of utilizing MMPs produced by other cells including tumor stromal cells $(16,20,21)$. Thus, the hypoxic tumor cells apart from stromal cells can participate in the invasive process since these hypoxic cells produce some bioactive proteins that act as a paracrine factor. Further studies are warranted to clarify the dynamics of microenvironmental interaction between tumor cells and surrounding stromal cells in OSCC.

\section{Acknowledgements}

The authors are indebted to Ms. K. Takahashi and Mr. K. Kinjyo for their excellent technical assistance. 


\section{References}

1. Landis SH, Murray T, Bolden S and Wingo PA: Cancer statistics, 1999. CA Cancer J Clin 49: 8-31, 1999.

2. Myoung H, Kim MJ, Hong SD, Lee JI, Lim CY and Hong SP: Expression of membrane type I-matrix metalloproteinase in oral squamous cell carcinoma. Cancer Lett 185: 201-209, 2002.

3. Kato K, Hara A, Kuno T, Mori H, Yamashita T, Toida $M$ and Shibata T: Aberrant promoter hypermethylation of p16 and MGMT genes in oral squamous cell carcinomas and the surrounding normal mucosa. J Cancer Res Clin Oncol 132: 735-743, 2006

4. Quintero M, Mackenzie N and Brennan PA: Hypoxia-inducible factor 1 (HIF-1) in cancer. Eur J Surg Oncol 30: 465-468, 2004.

5. Semenza GL: HIF-1, O(2), and the 3 PHDs: how animal cells signal hypoxia to the nucleus. Cell 107: 1-3, 2001.

6. Semenza GL: Targeting HIF-1 for cancer therapy. Nat Rev Cancer 3: 721-732, 2003.

7. Sasabe E, Tatemoto Y, Li D, Yamamoto T and Osaki T: Mechanism of HIF-1alpha-dependent suppression of hypoxiainduced apoptosis in squamous cell carcinoma cells. Cancer Sci 96: 394-402, 2005.

8. Gu J, Milligan J and Huang LE: Molecular mechanism of hypoxia-inducible factor 1alpha-p300 interaction. A leucinerich interface regulated by a single cysteine. J Biol Chem 276: 3550-3554, 2001

9. Rijken PF, Bernsen HJ, Peters JP, Hodgkiss RJ, Raleigh JA and van der Kogel AJ: Spatial relationship between hypoxia and the (perfused) vascular network in a human glioma xenograft: a quantitative multi-parameter analysis. Int J Radiat Oncol Biol Phys 48: 571-582, 2000.

10. Kaanders JH, Wijffels KI, Marres HA, Ljungkvist AS, Pop LA, van den Hoogen FJ, De Wilde PC, Bussink J, Raleigh JA and van der Kogel AJ: Pimonidazole binding and tumor vascularity predict for treatment outcome in head and neck cancer. Cancer Res 62: 7066-7074, 2002.
11. Liotta LA, Steeg PS and Stetler-Stevenson WG: Cancer metastasis and angiogenesis: an imbalance of positive and negative regulation. Cell 64: 327-336, 1991.

12. Miyoshi A, Kitajima Y, Ide T, Ohtaka K, Nagasawa H, Uto Y, Hori $\mathrm{H}$ and Miyazaki K: Hypoxia accelerates cancer invasion of hepatoma cells by upregulating MMP expression in an HIF-1alpha-independent manner. Int J Oncol 29: 1533-1539, 2006.

13. Semenza GL: HIF-1 and mechanisms of hypoxia sensing. Curr Opin Cell Biol 13: 167-171, 2001.

14. Bussink J, Kaanders JH and van der Kogel AJ: Tumor hypoxia at the micro-regional level: clinical relevance and predictive value of exogenous and endogenous hypoxic cell markers. Radiother Oncol 67: 3-15, 2003.

15. Arteel GE, Thurman RG, Yates JM and Raleigh JA: Evidence that hypoxia markers detect oxygen gradients in liver: pimonidazole and retrograde perfusion of rat liver. Br J Cancer 72: 889-895, 1995.

16. Deryugina EI and Quigley JP: Matrix metalloproteinases and tumor metastasis. Cancer Metastasis Rev 25: 9-34, 2006.

17. Munoz-Najar UM, Neurath KM, Vumbaca F and Claffey KP: Hypoxia stimulates breast carcinoma cell invasion through MT1-MMP and MMP-2 activation. Oncogene 25: 2379-2392, 2006.

18. Semenza GL: Hypoxia-inducible factor 1: oxygen homeostasis and disease pathophysiology. Trends Mol Med 7: 345-350, 2001.

19. Fujiwara S, Nakagawa K, Harada H, Nagato S, Furukawa K, Teraoka M, Seno T, Oka K, Iwata S and Ohnishi T: Silencing hypoxia-inducible factor-1alpha inhibits cell migration and invasion under hypoxic environment in malignant gliomas. Int $\mathbf{J}$ Oncol 30: 793-802, 2007

20. Kato K, Hara A, Kuno T, Kitaori N, Huilan Z, Mori H, Toida M and Shibata T: Matrix metalloproteinases 2 and 9 in oral squamous cell carcinomas: manifestation and localization of their activity. J Cancer Res Clin Oncol 131: 340-346, 2005.

21. Thomas GT, Lewis MP and Speight PM: Matrix metalloproteinases and oral cancer. Oral Oncol 35: 227-233, 1999. 